\title{
Recommendations for translation and cross-cultural adaptation of instruments for occupational therapy research and practice
}

\section{Recomendações para a tradução e adaptação transcultural de instrumentos para a pesquisa e a prática em Terapia Ocupacional}

\author{
Wendy J. Coster ${ }^{1}$, Marisa C. Mancini \\ http://dx.doi.org/10.11606/issn.2238-6149.v26i1p50-7
}

Coster WJ, Mancini MC. Recommendations for translation and cross-cultural adaptation of instruments for occupational therapy research and practice. Rev Ter Ocup Univ São Paulo. 2015 jan./ abr.;26(1):50-7.

ABSTRACT: Much of the work developing and testing new instruments for use in rehabilitation research and practice has occurred in English-speaking countries. Therefore rehabilitation researchers and clinicians in other countries who want to use these instruments must first undertake the work of translating them into their own language and then validating their use in a different cultural context. This process must follow established guidelines in order for the translated instrument to be valid for use. To date, however, there has been little discussion in the occupational therapy literature about how best to conduct translation or how to evaluate the validity of a translated instrument. The purpose of this article is to provide guidance to readers who may be considering translation of an instrument for occupational therapy research or practice and to assist practitioners who are evaluating whether they should use a translated instrument in their clinical work.

KEYWORDS: Measurement; Translation; Cross-cultural.
Coster WJ, Mancini MC. Recomendações para a tradução e adaptação transcultural de instrumentos para a pesquisa e a prática em Terapia Ocupacional. Rev Ter Ocup Univ São Paulo. 2015 jan./abr.;26(1):50-7.

RESUMO: Grande parte do desenvolvimento e validação de novos instrumentos para o uso na pesquisa e na prática da reabilitação ocorreu em países de língua inglesa. Assim, pesquisadores e profissionais da reabilitação em outros países precisam, inicialmente, assumir a tarefa de traduzir tais instrumentos para outros idiomas e validar seu uso em um contexto cultural diferente. É necessário que esse processo siga diretrizes sólidas para que o instrumento traduzido possa ser validado para uso. No entanto, há poucas discussões na literatura em Terapia Ocupacional sobre como melhor conduzir a tradução ou como avaliar a validade de um instrumento traduzido. O objetivo deste artigo é oferecer orientações aos leitores que estejam considerando traduzir um instrumento para a pesquisa ou a prática em Terapia Ocupacional e auxiliar os profissionais a avaliarem o uso de instrumentos traduzidos em sua prática clínica.

DESCRITORES: Mensuração; Ttradução; Transcultural.

1. Department of Occupational Therapy. Boston University.

2. Department of Occupational Therapy. Universidade Federal de Minas Gerais.

Corresponding author: Wendy Coster, PhD, OTR/L. Department of Occupational Therapy. College of Health and Rehabilitation Sciences: Sargent College. Boston University. 635 Commonwealth Avenue. Boston, MA 02215 - USA. E-mail: wjcoster@bu.edu. 
$\mathrm{R}$ ecent years have seen tremendous growth in the development of instruments for use in rehabilitation research and practice, particularly instruments that focus on activity and participation. In part this emerging focus reflects increasing recognition that without appropriate measures, even the best-designed clinical trial cannot provide valid information about treatment effectiveness or the long-term outcomes of individuals with disabilities ${ }^{1}$. Many older instruments were not designed for these purposes and either do not address the daily life concerns of individual with disabilities, or do so in ways that are biased or uninformative for treatment planning.

Much of the work developing and testing new instruments has occurred in English-speaking countries, including Canada (e.g., Canadian Occupational Performance Measure) ${ }^{2}$, the United States (e.g., Stroke Impact Scale) ${ }^{3}$, Australia (e.g., Melbourne Assessment of Unilateral Upper Limb Function) ${ }^{4}$, and the United Kingdom (e.g., RolandMorris Questionnaire) $)^{5}$. Therefore rehabilitation researchers and clinicians in other countries who want to use these instruments must first undertake the work of translating them into their own language and then validating their use in a different cultural context. This process is neither quick nor simple, and must follow established guidelines in order for the translated instrument to be valid for use. To date, however, there has been little discussion in the occupational therapy literature about how best to conduct translation or how to evaluate the validity of a translated instrument. Accordingly, the purpose of this article is to provide guidance to readers who may be considering translation of an instrument for occupational therapy research or practice and to assist practitioners who are evaluating whether they should use a translated instrument in their practice.

\section{General Considerations}

Before undertaking a translation two important questions need to be examined carefully. The first question is whether the instrument is really needed and why. Translation and cross-cultural validation require substantial resources and effort and not all the instruments being used elsewhere are necessarily a good investment of these resources. A good way for translators to think about this question is to write out what important research or practice question the translated instrument would enable them to address and why currently available tools are inadequate for this purpose. In other words, translators should have a clear idea of their specific purpose for the instrument and the context they want to use it in. An example of this kind of statement might be: "This new instrument includes questions about the person's social participation that are not included in any other available instrument. Therefore we have no way to collect systematic data about the social participation of people with multiple sclerosis."

A second related question is whether the instrument proposed for translation is really the best instrument to use for that purpose. In the context of translation, this means: is the instrument well suited to the intended purpose, population, and clinical context? This question can't be answered without a careful review of the content, including the rating or scoring criteria, and the literature that has been published on the original instrument. If an instrument contains many items that refer to daily activities or use of objects and equipment that are not common in the translation culture, this may raise doubts about its cross-cultural relevance. For example, suppose a developmental assessment of children involves use of toys that are not available in the translation culture. Although the instructions to the child can be translated into their language, their performance with the objects cannot validly be compared to that of children who have had years of experience with the toys in the instrument's original culture. In other words, the translated instrument will not be measuring the same ability (See Table 1 for some examples). A different instrument that allows more flexibility in choice of objects may be a better focus for translation because it might allow the users to substitute objects that are familiar in the local culture.

Table 1 - Examples of items from US instruments that may be culturally inappropriate in other countries

\begin{tabular}{l|l}
\hline \multicolumn{1}{c|}{ Item } & \multicolumn{1}{c}{ Issue } \\
\hline Getting in and out of a bathtub & Homes in many countries only have a shower, not a tub \\
\hline Doing snow sports & Not appropriate in countries that do not have cold winters \\
\hline $\begin{array}{l}\text { Participating in school-sponsored teams, clubs and } \\
\text { organizations }\end{array}$ & $\begin{array}{l}\text { In many countries, teams and clubs are sponsored by community groups, } \\
\text { not by schools }\end{array}$ \\
\hline Going up and down an escalator & Will only be found in urban, developed areas \\
\hline
\end{tabular}


Another example involves items that ask the client to report on his or her attitudes, beliefs, or emotional states. Several issues should be considered. The first issue is whether the concepts referred to in the items exist within the translation culture. For example, concepts such as empowerment are prominent in the English-language rehabilitation literature, but may not make sense to clients in a culture that values inter-dependence over individual autonomy. Cultures also have different standards for what is an acceptable topic for conversation. This could create difficulties if an instrument that is going to be used in an interview context contains many items that would be considered inappropriate or even offensive (e.g., questions about income or sexual behavior) (See Herdman et al. ${ }^{6}$ for a detailed discussion).

Finally, it is important to examine the psychometric evidence to determine whether it supports the use of the instrument for the translator's purpose and context. In addition to examining the standard criteria of reliability coefficients and validity evidence, if the translator intends to use the instrument to examine change related to treatment, then evidence of the sensitivity or responsiveness to change will be important ${ }^{7}$.

In the next section, we review the series of steps involved in translation with examples to illustrate some of the issues that may arise. Five important steps will be discussed: 1) Permission; 2) translation and reconciliation; 3) back-translation and reconciliation; 4) review of final version; 5) evaluation.

\section{Step 1: Secure Permission}

Before any translation work can begin permission needs to be obtained from the appropriate source. If the instrument is published commercially (i.e., by a publishing company), then the publisher, not the author, should be contacted because the publisher holds the copyright. Otherwise, the primary author (usually identified on the instrument score form or in the major published papers) is the person to contact. The first contact serves two purposes: first, to find out whether someone else has already begun a translation in the country and language of interest; and second, to find out what the author's or publisher's requirements are for an agreement to translate. Most authors and publishers require some form of written protocol from the translator that describes who will manage the project, who will do the translation (e.g., documentation of the translator's expertise in both languages), what components of the instrument will be translated (e.g., items, score form, manual, report form) and what steps will be followed. Other important questions also need to be discussed between the author/publisher and translator. Will any form of cultural adaptation be permitted? The answer to this question may depend on the type of instrument. Functional assessments may allow more variation since they are intended to assess the person's performance in his or her current context. Who will publish and distribute the translated instrument? Some publishers want to retain all control over the distribution (and profit from sales) of a translated instrument. On paper-and-pencil instruments that can be photocopied, the author/publisher may have certain information about authorship and copyright that must be included in a specific format. Will there be any restrictions on who can use the translated instrument? In some situations a publisher may grant permission for research use of a translated version, but not for general use in clinical practice. A sample set of guidelines is included in Appendix A to illustrate some of the questions that potential translators should consider. The translator should also ask whether the author or publisher's representative will be available for consultation about any questions or issues that might come up during the translation process.

Based on conversations with and guidelines provided by the author/publisher, the person heading the translation effort prepares a final protocol for the proposed translation process. This step of the process is completed when the author/publisher and the person directing the translation have reached agreement on these issues and the agreement has been documented in writing.

\section{Step 2: Translation}

Up to this point we have referred to "the translator", but in reality translation should be a collaborative team effort. Translation of material from one language to another is never a straightforward process: there may be multiple options for how to translate a particular word or phrase or there may be no exactly equivalent translation for a particular term. Different translators may make different choices, therefore recommended translation procedures involve replication at multiple points. In the same way that averaging results of several trials of grip strength or walking speed gives a more reliable estimate of a person's true ability, comparison of two different translations helps to ensure the final version is as accurate as possible. The initial translation should always be done by at least two people who work independently. Alternatively, two teams of 2-4 people each may complete the initial translation. It is important that the two translators or teams do not communicate about their work until the translation is complete so that results can 
be compared. In addition to the translators, another person with fluency in the foreign language should function as the manager of the translation process.

The most obvious qualification for a translator is fluency in both the original language and the translation language. Published guidelines recommend that the translators' native language be the language they are translating into $^{8.9}$. However familiarity with the field the instrument is designed for as well as the instrument itself is also important. For example occupational therapy, rehabilitation, and medicine use terms with particular meanings that are well understood by practitioners in the field, but may not be clear to persons outside the field. This specialized knowledge may be helpful in deciding among several possible options for translating a word or phrase. Familiarity with the instrument helps the translator understand what the intent of a particular item or rating criterion is so that the equivalent meaning is used in translation. Careful reading of the instrument's manual and related articles before beginning translation helps build this understanding.

Once the two independent translations have been completed, they should be given to the manager of the translation process, who then initiates a reconciliation process. This step involves comparison of the two translations to identify points of difference and then reconciling the differences to create a single version. This process can be carried out in a variety of ways depending on the team. One approach is to have the members of both teams, along with the manager, review and discuss the discrepancies together to arrive at a consensus on each point.

If the author of the instrument has agreed to be involved, points where the group cannot agree can be brought to the author to obtain more information about the intent of the original version. This additional information may help the team determine the most appropriate translation. If the team is unable to reach consensus with these methods, they may need to bring in additional reviewers to help resolve the difference. Obviously it is ideal if these outside reviewers have similar backgrounds in terms of language skills and professional knowledge as the original translators.

When a final consensus version of the translated instrument has been developed, it is ready for the backtranslation process.

\section{Step 3: Back translation}

Back translation is a check on the accuracy of the translation. In this step, the translated instrument is translated back into the original language and then compared to the original version. The back translation should be done by members of the team who were not involved in the original translation effort so that they will be unbiased. Similar to the original translation process, it is recommended that two individuals, or two small groups, complete independent back translations, which can then be compared. If possible, at least one of the translators should be a person for whom English is the first language.

When the back translations are complete, the translation team manager compares the two versions to identify discrepancies between them and between the back-translations and the original instrument. There are several approaches to deal with the discrepancies that may be found. Usually small grammatical differences can be ignored (unless language skill is the focus of the assessment). Differences between the back-translation and original version that reflect differences in meaning are more important. To resolve these bigger issues, the translation manager and back translators can meet to discuss the differences and see whether, through discussion, they can come to agreement on a better translation. Differences in back translation may simply reflect different ways of expressing certain meanings, and therefore it may not be difficult to reconcile the original and translated instruments. If agreement is difficult to reach, it may be important to consult additional bi-lingual experts to advise on the proper interpretation and translation of the English phrasing.

It is very important that translation and back translation are done for all portions of the instrument that may affect administration and scoring. Thus, not just the items but also the scoring or rating descriptions need to undergo this process, as well as instructions for administration and scoring. Inappropriate variations in any of these aspects of the instrument could affect the crosscultural validity of the translated version.

After agreement has been achieved between the translation, back translation, and original version, two additional steps are very helpful. The first step is to have the translated version reviewed by a new group of potential users of the instrument such as other clinicians who were not involved in the translation. The purpose of this external review is to identify places where meaning is unclear or wording is perceived as awkward or unfamiliar. In large countries such as Brazil, it may be important to recruit reviewers from several different regions who can determine whether the language used is consistent with language usage in their region.

A second step, known as "cognitive testing" or "cognitive interviewing", is particularly important for self-report or interview instruments. This process involves 
administering the translated instrument to members of the population for whom it was intended and exploring whether they understand the items as intended. For example, a parent might be asked to complete a measure of their child's daily life skills. After reading each question, the interviewer asks the parent to "talk out loud" as he or she decides what the item means and what the appropriate answer for their child would be. In this way the interviewer can tell immediately if the parent finds the item confusing, doesn't understand some of the words, or can't decide how to select the appropriate answer. By listening carefully to the parent, the interviewer may also be able to identify ways to improve the wording. The translation team may do two or three interviews, then make changes that seem to be needed, and then conduct another two to three interviews to see if the changes have corrected the problems (See Collins ${ }^{10}$ and Streiner and Norman ${ }^{11}$ for additional details). When making changes, the team should be careful to make sure that the original meaning of the item isn't changed.

\section{Step 4: Development of final version}

Usually the last stage of the translation process involves review with the original author and/or publisher of the instrument, who will check to make sure that the translation is accurate and maintains the important features of the original instrument. They will use the back-translated version of the instrument for this purpose. The optimal situation is when the author can collaborate directly with the translation team on this review. For example, if the author finds phrasing in the back-translation that isn't identical to the original version, he or she can talk to the team to determine whether the translation phrasing retains the intended meaning in the translation language. If it isn't quite right, the author can help the team to understand the meaning of the English term, and then to find the best equivalent term in the translation language.

\section{Step 5: Evaluation of the translated instrument}

Achieving an approved translation is a big accomplishment. Yet, before the instrument can be used with confidence in research and practice, it needs to be evaluated like any other new instrument. The larger the differences in language and culture between the country where the instrument was originally developed and the country of translation, the more critical this evaluation is. However, even cultures that share many similarities in terms of level of technological development, social organization, or values may differ in ways that could affect how people respond to the instrument. These differences could affect both reliability and validity.

At a minimum, information about the internal consistency of the scales and the stability of scores across occasions (test-retest) and/or raters (inter-rater) is needed. This information is used as evidence that the items appear to be measuring a common underlying construct and that scores from a single occasion or rater provide a sound estimate of the person's typical performance, disability experience, emotional state, or beliefs. This information is also needed to estimate the standard error of measurement (SEM) so that users can determine when change over time is greater than measurement error ${ }^{11}$.

Priorities for additional evaluation depend on the nature of the instrument and the immediate needs of likely users. For example, if the instrument is going to be used for developmental assessment of children, then normative data from the translation country will need to be collected since it can't be assumed that norms from the country of origin will be appropriate. On the other hand, if the instrument is going to be used to assess disability, then research to examine whether scores from the translated instrument correlate as expected with other disability measures currently in use will be needed. If the instrument has several subscales that were based on a factor analysis, then the factor analysis will need to be replicated using data obtained with the translated version to determine whether that structure is maintained. Finally, if the instrument was developed using Rasch or IRT methods, these analyses should be repeated on a sample from the translation country, including analysis of item fit and item difficulty order, as well as comparison of item difficulty estimates to evaluate whether there are significant differences between the two versions (differential item function, or DIF)?

\section{Considerations of cultural equivalence}

At the beginning of this paper the question of crosscultural equivalence was introduced when it was advised to review the instrument for its cultural relevance before beginning the translation process. However, questions about cultural relevance can appear at any point in the translation process, bringing, in turn, questions about how to resolve these issues. For example, an item in a self-report of daily activities may be readily translatable, but one or more of the examples used for illustration might not be appropriate (e.g., an item on eating utensils uses the example of fork and knife, not chopsticks). This is one of the situations where discussion with the author may be especially important because the author will need to determine whether changing 
the examples is a significant change in the item or which of several substitute examples best retains the original meaning of the item.

Other issues may be more challenging. For example, in some cultures where services are available to most people with disabilities, the users of these services may be familiar with terms such as "barriers" or "supports" or "accessibility". Although it may be possible to find a literal translation of such terms, those terms may be unfamiliar and even off-putting to clients. Is it acceptable to substitute different phrasing that is more familiar to potential respondents to the instrument? Such an alteration may improve local validity, but may not make the translated instrument completely comparable to the original. This is another example where discussion with the author may be critical to finding an appropriate solution. Some authors are quite firm that no changes can be made to the original instrument, in which case the translation team will need to decide whether their purpose can really be achieved through translation, If not, the team will need to find or develop an alternative instrument.

\section{CONCLUSION}

Translation of existing instruments has many advantages over developing a completely new instrument. Usually there is an existing body of research that supports the reliability of the scores and their validity for particular purposes. In addition, prior users of the instrument may have reported on particular situations where it has been most helpful. The availability of this information can help the potential translator make informed choices about whether to proceed with translation or not. Use of translated instruments also helps to connect research findings from different countries and to identify how outcomes are similar and different across various regions of the world ${ }^{12}$. Capitalizing on these advantages, however, depends on a careful and systematic translation effort.

\section{Appendix A. Example of Guidelines for Translation and Cross-cultural Adaptation of an Instrument}

Thank you for taking the time to read our guidelines and for proposing to translate the INSTRUMENT into your own language. Knowledge translation and the sharing of information is a vital part of the work we do.

Developing the INSTRUMENT for another language will require translation of the following:

- Items and scoring criteria;

- administration guide;

- score reporting forms;

- Manual.

You must be sure you have the resources to cover all of these costs of translation. The authors of the INSTRUMENT require documentation that these resources will be available to the person(s) proposing to make a translation before granting approval. The steps for conducting an approved translation of INSTRUMENT are outlined below.

1. Before starting to translate any portion of the INSTRUMENT, contact us at (EMAIL) to obtain approval to proceed. We will check to make sure that a similar translation is not already in progress and that you have the necessary resources to carry out the project. You will need to submit a description of the translation and adaptation process you propose to follow that also includes:

- The name and credentials (degrees and other evidence of expertise in English translation) of the persons who will be conducting the translation;

- Names or description of potential users who will review the translation;

- Name and credentials of the person(s) who will complete the back translation.

- Resources available to support the costs of translation and adaptation.

Please do not proceed until you have a signed agreement from us.

Please ensure that you retain all the content of the English version of the INSTRUMENT in your translation, including: copyright information, acknowledgement of original authors, website address, manual, etc.

2. As you proceed with translating the INSTRUMENT items into your language of choice, please contact (AUTHOR) when in need of clarification. 
Please remember that effective translations also need to take into account the customs and culture of your region to reflect accurately the intention of the wording in the original English version. Some words do not directly translate and there may need to be discussion with AUTHOR to make sure that the correct meaning is retained in the translation.

3. As part of the translation process, there must be ongoing communication in the form of brief status updates (e.g., stage of translation in progress, projected date of completion, etc.) over the duration of the translation, which we initially determine to be a 1-year period. These status updates are to be sent to AUTHOR and will be required at 4-month intervals from the date of approval of your intent to translate. If you require additional time to complete the translation, you may apply to us for an extension.

4. In your translated version, please include information about the person or organization that translated the document (including name and e-mail address) and any other acknowledgements you would like to make.

5. When your DRAFT translation is ready, have another person who is fluent in both English and your own language translate your version back into English (i.e., back- translation). Then forward an electronic version of this 'back translated' English copy and the translated copy to AUTHOR so that we can review it.

6. When translation steps listed above have been completed and the translated version has been approved, a Word or Excel copy of the final version of all materials should be forwarded to us for our records. We also will need the name and e-mail address of the person to whom any questions about the translation should be directed.

7. The translation team may submit up to 3 names of key members of the team who will be granted permission to use the translated version of the INSTRUMENT for their research activities at no cost to them.

8. PUBLISHER will make the translated instrument available for purchase on its website. The translation team will receive a royalty of PERCENTAGE (\%) of the sales of the translated version of the INSTRUMENT in the home country for a period of 5 years.

\section{REFERENCES}

1. Coster WJ. Making the best match: selecting outcome measures for clinical trials and outcome studies. Am J Occup Ther. 2013;67:162-70. doi: 10.5014/ajot.2013.006015.

2. Law M, Baptiste S, Carswell A, McColl MA, Polatajko H, Pollock N. Canadian Occupational Performance Measure. Ottawa: CAOT Publications; 2005.

3. Duncan PW, Wallace D, Lai SM, Johnson D, Embretson S, Laster L J. The stroke impact scale version 2.0. Evaluation of reliability, validity, and sensitivity to change. Stroke.1999; 30:2131-40. doi: 10.1161/01.STR.30.10.2131.

4. Randall M, Carlin JB, Chondros P, Reddihough D. Reliability of the Melbourne Assessment of Unilateral Upper Limb Function. Dev Med Child Neurol. 2001;43:761-7. doi: 10.1111/j.1469-8749.2001.tb00158.x

5. Roland M, Morris R. A study of the natural history of back pain. Part 1: development of a reliable and sensitive measure of disability in low-back pain. Spine. 1983; 8:141-4.

6. Herdman M, Fox-Rushby J, Badia X. A model of equivalence in the cultural adaptation of HRQoL instruments: the universalist approach. Qual Life Res. 1998;7:323-35. Available from: http://goo.gl/S73e $7 \mathrm{~K}$.

7. Mokkink LB, Terwee CB, Knol DL, Stratford PW, Alonso J, Patrick DL, Bouter L, de Vet HCW. The COSMIN checklist for evaluating the methodological quality of studies on measurement properties: a clarification of its content. BMC Med Res Methodol. 2010; 10:22-30. doi: 10.1186/14712288-10-22.

8. Beaton DE, Bombardier C, Guillemin F, FerrazMB. Guidelines for the process of cross-cultural adaptation of self-report measures. Spine. 2000;23:3186-91. Available from: http:// goo.gl/LF9zgR.

9. Wild D, Grove A, Martin M, Eremenco S, McElroy S, VerjeeLorenz A, Erikson P. Principles of good practice for the translation and cultural adaptation process for patient-reported outcomes (PRO) measures: Report of the ISPOR task force for translation and cultural adaptation. Val Health. 2005;8:94-104. doi: 10.1111/j.1524-4733.2005.04054.x 
Coster WJ, Mancini MC. Recommendations for translation and cross-cultural. Rev Ter Ocup Univ São Paulo. 2015 jan./abr.;26(1):50-7.

10. Collins D. Pretesting survey instruments: an overview of cognitive methods. Qual Life Res. 2003;12:229-38. Available from: http://goo.gl/IhZRM4.

11. Streiner DL, Norman GR. Health measurement scales: a practical guide to their development and use. 4th ed. Oxford: Oxford University Press; 2008.

Recebido para publicação: 01/09/2014

Aceito para publicação/tradução: 15/12/2015
12. Haley SM, Coster WJ, Kao YC, Dumas HM, Fragala-Pinkham MA, Kramer JM, Ludlow LH, Moed R. Lessons from use of the Pediatric Evaluation of Disability Inventory: Where do we go from here? Pediatr Phys Ther. 2010;22:69-75. doi: 10.1097/ PEP.0b013e3181cbfbf6. 